Original Research Paper

\title{
The Extract Region of Interest in High-Resolution Palmprint using 2D Image Histogram Entropy Function
}

\author{
${ }^{1}$ Inass Shahadha Hussein, ${ }^{1}$ Shamsul Bin Sahibuddin, ${ }^{2}$ Md Jan Nordin and ${ }^{1}$ Nilam Nur Amir Sjarif \\ ${ }^{I}$ Faculty Razak of Technology and Informatics, UTM, Kuala Lumpur, Malaysia \\ ${ }^{2}$ Faculty of Information Science and Technology, UKM, Bangi, Malaysia
}

\author{
Article history \\ Received: 02-01-2019 \\ Revised: 06-03-2019 \\ Accepted: 07-05-2019 \\ Corresponding Author: \\ Inass Shahadha Hussein \\ Faculty Razak of Technology \\ and Informatics, UTM, Kuala \\ Lumpur, Malaysia \\ Email: inasshussin@yahoo.com
}

\begin{abstract}
The segmentation of high-resolution palmprint is has been challenged and the research in this filed is still limited because of variations in location and distortion of these images. To achieve superior recognition result, accurate segmentation of a region of interest is very crucial. Therefore, in this paper, a novel palmprint extraction method has been presented using a 2D image histogram entropy function and mathematical dilation. The proposed method has two phases. The first phase is the binarization image where the histogram of the image will be determined after applying a median filter to remove noise and then calculating the 2D image histogram entropy function. Finally, the maximum entropy that will be the adaptive threshold value to build a binary palmprint image will be selected. The second phase is to extract the ROI, apply a dilation method on the binary image, then dividing the dilate image into four regions and finding four reference points depending on the white percentage and finally the ROI will be extracted. The publically available high-resolution palmprint THUPALMLAB has been used for testing. The result indicates a high percentage of accuracy up to $93 \%$. The findings strongly indicate that the proposed method was able to extract the palm's ROI more consistently. These ROIs will be used in the recognition system instead of whole palmprints and hence assists in improving the performance of a traditional palmprint system. High-resolution palmprint images are highly used in the forensic application.
\end{abstract}

Keywords: ROI, High-Resolution Palmprint, Histogram, Entropy, Dilation

\section{Introduction}

Palmprint biometric system is deemed as a promising system in identifying methods of authentication, in both civil and forensic applications. Palmprint image can be classified into low-resolution (below 150 ppi) and highresolution (about 500 ppi). Low resolution is appropriate for commercial and civil applications while high resolution is used for forensic applications (Jain and Feng, 2009; Hussein and Nordin, 2014; Fei et al., 2018; Jamshidnezhad et al., 2012). In addition, it is important to note that any biometric system consists of four major steps: Image capture, image processing (which includes image segmentation), feature extraction and matching (Kumar, 2018; Wu et al., 2018; Zhang et al., 2018;
Kahaki et al., 2016; Ashtari et al., 2015). The segmentation of an image to extract the ROI is considered a major step in a biometric system. The accuracy of the recognition algorithms entirely depends on harmonious segmentation. The literature indicates that the generality of the low-resolution palmprint extraction ROI algorithms shares a view that the placement of the segmented square area needs to include these main steps. The first step is binarizing the image which is located at the two key points in the gaps between the fingers. In the second step, the ROI is detected as a square region (Kim, 2011; Jaswal et al., 2017; Zhang et al., 2017). Figure 1 shows an example of a lowresolution ROI which includes the most significant features; principle lines, wrinkles and ridges. 
Nevertheless, these algorithms are not appropriate for high-resolution images. The problems of a highresolution image are mainly caused by the variation in the location and the distortion of the skin images (Carreira et al., 2014). Therefore, the problem made it difficult to extract ROI. Figure 2 illustrates a highresolution image with main features; ridges, creases and minutiae points. To the best of our knowledge, these problems have yet to be solved, compounded by the fact that only a few researchers have attempted segmenting the high-resolution image. Instead, some of the researchers had tried to enhance the image without segmenting it (Mousavi Kahaki et al., 2016). Jain and Feng (2009) introduced the high-resolution palmprint image enhancement by separating the seed selected region, into creases and ridges. He then smoothed the ridge's direction using a Gabor filter known as the region grown algorithm until the image is enhanced. Next, this algorithm was evaluated using 150 live-scans and 100 latent palmprints.

Carreira et al. (2014) proposed an image transformation of the blurred image using the motion blur inspired by the works of (Brusius et al., 2011). This resulted in a blurred image with a set of parallel stripes. These strips were detected using a directional filter followed by the construction of a low-pass pyramid image. This striped image is then binarized by thresholding it by adjusting the distance between the stripe blur image that was reconstructed. The authors used the THUPALMLAB database to evaluate their work. This work was deemed appropriate for full palmprint images but the distortion of the images was not discussed.

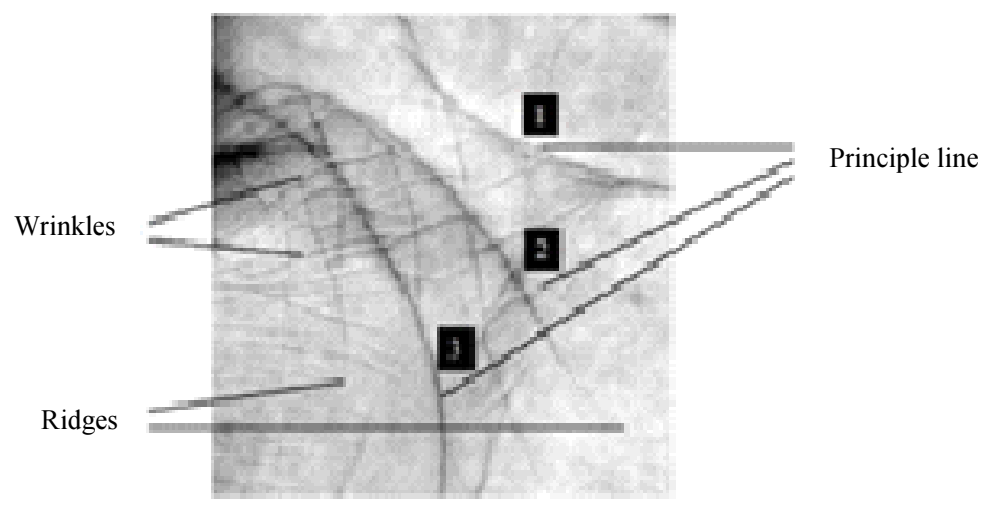

Fig. 1: ROI low-resolution palmprint

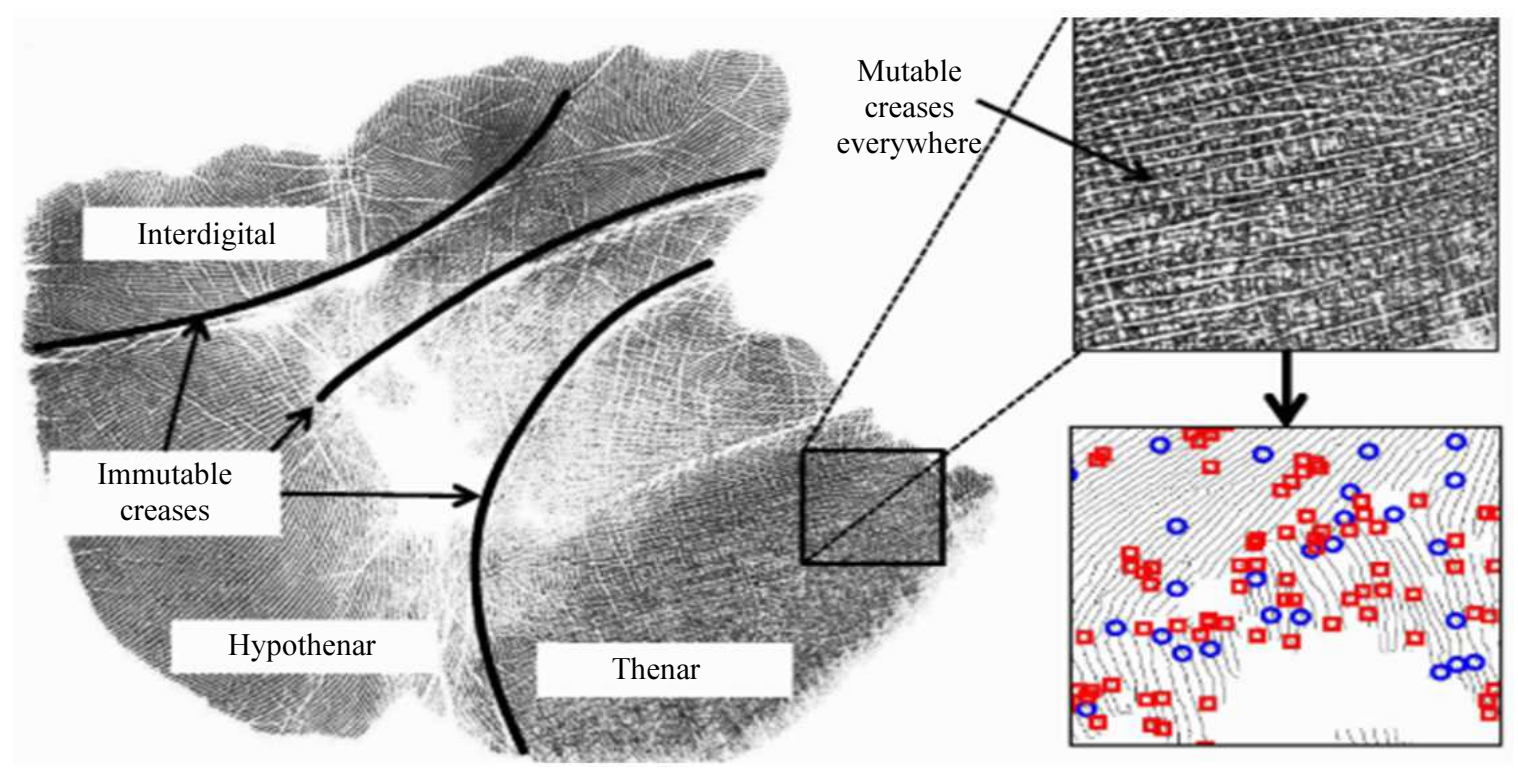

Fig. 2: High-resolution palmprint 
Wang et al. (2012) first introduced the latent to full palmprint based on radial triangulation by modifying the local minutiae points comparison. They then applied the comparison score using logistic regression learning. Subsequently, Wang et al. (2013a) introduced a method to automatically segment the region of the palmprint into three regions named as the interdigital, hypothenar and thenar using the datum point detection as illustrated by the following steps. First, apply a canny edge detector for all the palmprint images. Second, apply the first datum point using the convex hull to detect the endpoint of the heart line of the palmprint. Third, apply the second datum point to detect the end point of the lifeline of the palmprint. Four, the palmprint regions were then segmented according to the two datum points. Moreover, in the same year, Wang et al. (2013b) used these three regions which were segmented in the previous work to propose a matching strategy based on regional fusion, by first applying the region to region comparison and then the regional score level fusion based on the logistic regression. Later, (Wang et al., 2014) used these three regions to perform a regional fusion for the three region to region comparison scores using the spectral minutiae matching SMC. All these works were accomplished using the database obtained from THUPALMLAB. The methods constructed by Wang et al. (2014) produced a better result apart from solving the distortion problem.

The objective of this study is to segment the highresolution palmprint image and find the ROI, taking into consideration the distortion and the unallocated in these images. The main contribution of this study stems from the development of an accurate, high-resolution palmprint ROI extraction method by enhancing the image by applying the Median filter to remove the noise, calculate the 2D image histogram entropy function and select the maximum entropy to binarize the image. This is followed by applying a mathematical dilation and a novel approach so the ROI can be extracted. Figure 3 illustrates the block diagram of our proposed method, which will be explained in details in the following sections.

The rest of the paper is organized as follows. Section 2 described in detail the proposed method, followed by the experiment results in section 3. Finally, the paper is concluded in section 4 .

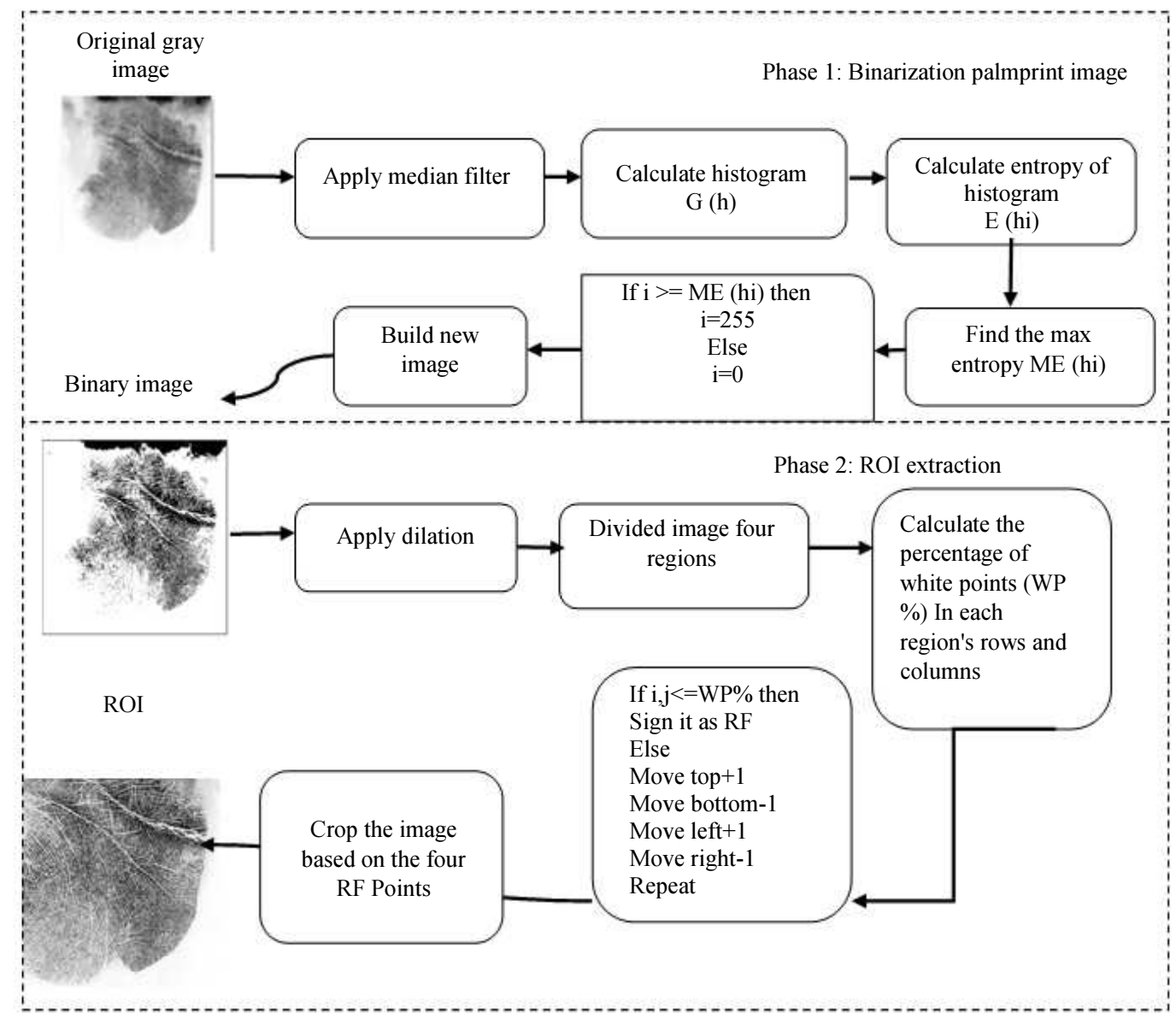

Fig. 3: Block diagram of the proposed method 


\section{Proposed Method}

In this section, we will explain in detail the proposed method that includes two phases which are the Binarization phase and the ROI extraction phase as illustrate in Fig. 3. In the following sub-sections, we will explain the steps involved in the proposed method.

\section{Binarization Phase}

The binarization phase has three steps. First, removing noise using the Median filter. Second, calculate the 2D palmprint image histogram and the third binarization of the palmprint image by calculating the 2D histogram entropy and select the maximum entropy that used as a threshold value.

\section{A. Remove Noise}

A digital filtering technique is often used to remove noise from an image. A median filter will be used in this situation. The noise in the palmprint image may occur during the collection of the image or as marks on the internal skin. The use of the median filter maintains the edge details of the palm, without substituting them with the non-reality values. It works by replacing the value of a pixel with the median pixels' neighborhood of the gray level. The median filter is represented by a square mask with $\mathrm{n} \times \mathrm{n}$ pixels where $\mathrm{n}$ must be an odd number (Mandeel et al., 2018). The median filter does a better job of removing the 'salt-and-pepper' noise, with less blurring of the edges. The steps of the Median filter algorithm are as follows:

1. Identify the neighborhoods and read all the intensity values within

2. Arrange the intensity values ascendancy

3. Specify the median among the values

4. Place the value at the center of the neighborhood

5. Repeat steps 1 to 4 to the end of the row until all the pixels are complete

The median filter is given by the following formula:

$m(x, y)=\operatorname{med}(f(x, y))$

where, $m(x, y)$ is the image after the determined median filter and $f(x, y)$ is the original image. In our proposed method we used a median filter $7 \times 7$. The use of the $7 \times 7$ median filter was proposed because of its effectiveness in removing the noise while maintaining the edge.

\section{B. 2D Palmprint Image Histogram}

A histogram statistically represents different pixels frequencies inside an image, the pixels that have various levels of intensity will represent by a 2D histogram. In this study, the $2 \mathrm{D}$ histogram of the gray palmprint image was calculated after removing the noise by applying a median filter in the according to the previous step. For a grayscale image with $8 \mathrm{bit} / \mathrm{pixel}$ and $I=255$, where $I$ is the highest intensity value, for each intensity level $\mathrm{i}$ $\in[0, \ldots \ldots \ldots, I]$. Gonzalez and Woods (2002) defined the formula of the histogram $h(i)$ image as:

$$
h(i)=n(i)
$$

where, $h(i)$ is the histogram of the gray level with the intensity $i, n(i)$ which is the number of pixels that have intensity $i$.

By applying Equation 2, the result is a 2D histogram with a 255 element integer array. This representation will require a complex calculation so a histogram normalization will be applied. This type of histogram will represent the distribution of the probability of various values of a pixel. This procedure is not difficult; a division of each 255 array elements of the histogram is made by the total number of all the pixels inside the image. Sometimes, the normalization is known as the contrast stretching or histogram stretching (Gonzalez and Woods, 2002). Figure 4 illustrates the histogram normalization while Fig. 5 shows the sample of the gray-level palmprint image histogram. Normalizing the histogram with the number of pixels in the image that leads to certain functions is called the Probability Density Function (PDF) of the levels of intensity which is given in the formula below (Gonzalez and Woods, 2002):

$$
p(i)=\frac{h(i)}{M \times N}
$$

PDF has the following properties:

$$
\left\{\begin{array}{c}
p(i) \geq 0 \\
\int_{-\infty}^{\infty} p(i)=1, \sum_{g=0}^{l} \frac{h(i)}{M \times N}=\frac{M \times N}{M \times N}=1
\end{array}\right.
$$

where, $h(i)$ is the histogram, $M \times N$ is the size of the image.

\section{Binarization based Maximum Entropy}

The image binarization methods are used to convert a grayscale image into a binary image. In the binarization process, each pixel in the grayscale image is examined by comparing its pixel value with a threshold value. A higher pixel value than the threshold leads to a pixel value of one (white) in the binary image and a lower value results in a pixel value of zero (black) (Kim et al., 2017). The researchers Sezgin and Sankur (2004) distinguished six different categories namely the thresholding algorithms based on the exploitation of (1) Histogram shape information, (2) Measurement space 
clustering, (3) Histogram entropy information, (4) Image attribute information, (5) Spatial information and (6) Local characteristics. This study adopted the 2D histogram entropy information as a novel approach to binarize the palmprint image in high resolution. The entropy-based approach is one of the most common techniques of image thresholding and it is widely used (Nie et al., 2017). The advantage of this algorithm is that it uses a global and objective property of the histogram due to its general nature if compared with other techniques. To begin with, the $2 \mathrm{D}$ palmprint image histogram entropy function is calculated as.

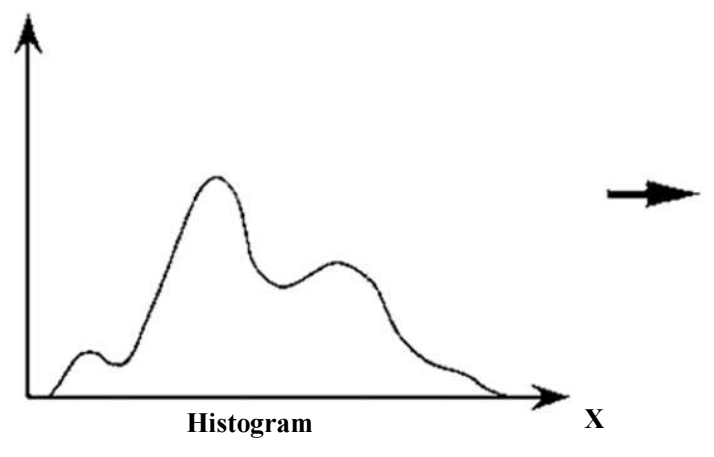

If the $h_{i}$ is the histogram of the image and by using various values between 0-256, the entropy function will be (Yang et al., 2018):

Where: $E(H)=-\sum_{i=0}^{l=1} p_{i} \log p_{i}$

where, $\quad p(i)=\frac{n_{i}}{(m \times n)}=$ probability in the gray level histogram where: $n_{i}$ a pixel in gray level, $0 \geq p_{i} \geq 1$.

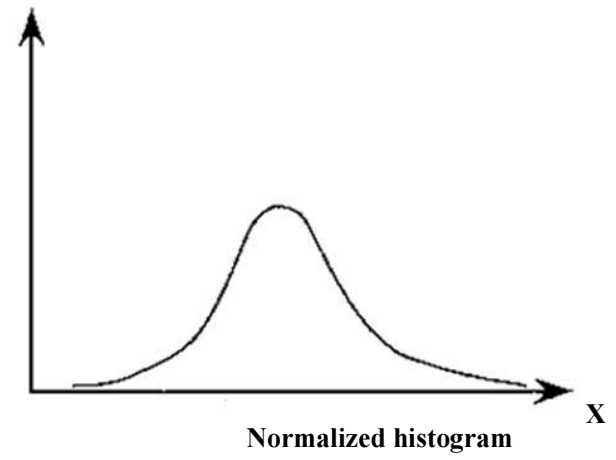

Fig. 4: Normalized histogram

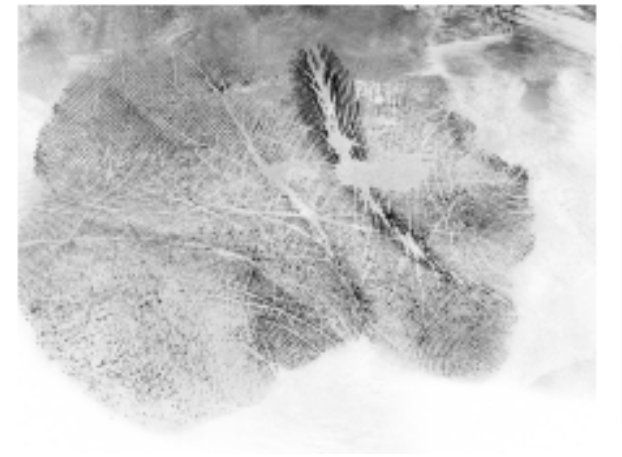

(a)

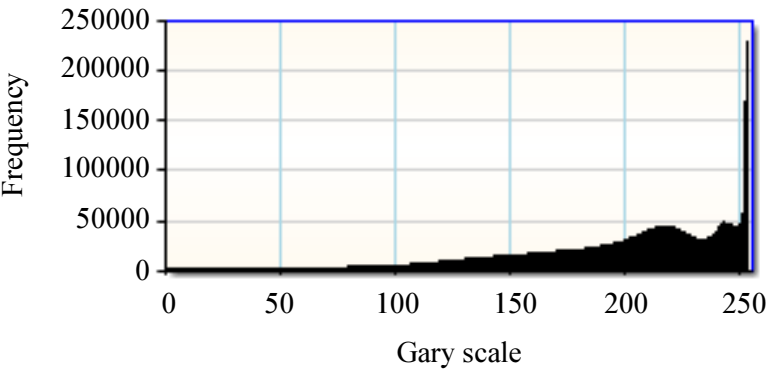

(b)

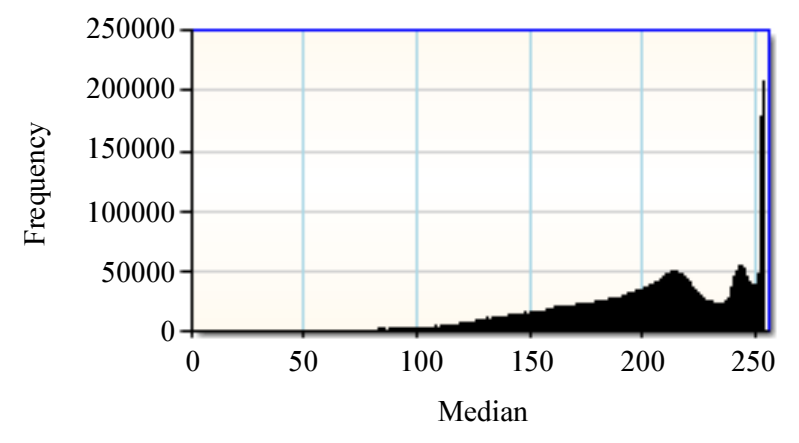

(c)

Fig. 5: Palmprint image histogram 
In Equation 4, the entropy is based on a $2 \mathrm{D}$ palmprint histogram specific for each column which corresponds with all the pixels' information and it will specify which pixel has the most information.

When the sign of the entropy value is mince, the entropy will be 0 ; that means the weak points in the image will be removed and force one will remain. This will act as filter work. After that, we select the maximum entropy value as an adaptive threshold for the image. The threshold value will compare with all the intensity in the histogram. If the intensity value is larger than the threshold value, the intensity will be 255 or else it will be 0 . The new histogram will be in 0 and 1 , which is used to build the new binary image. Figure 6 shows a sample of the calculation that the max entropy and the result binary palmprint image will be using in the next step as well as to extract the ROI. Figure 7 illustrates the algorithm steps of the binarization image based on the max entropy.

\section{ROI Extraction Phase}

After we obtained a binary palmprint image from the previous step, this image will feed the ROI extraction phase which includes two steps; applying the dilation and ROI construct.

\section{A.Morphology-Dilation}

Morphology is a broad set of image processing operations that process images based on shapes. Morphological operations apply a structuring element to an input image, creating an output image of the same size. In a morphological operation, the value of each pixel in the output image is based on a comparison of the corresponding pixel in the input image with its neighbors.

By choosing the size and shape of the neighborhood, a morphological operation that is sensitive to specific shapes in the input image can be constructed. The goal of morphological is to extract structural information (shape and size). The most basic morphological are dilation and erosion. Dilation adds pixels to the boundaries of objects in an image, while erosion removes pixels from object boundaries. The number of pixels added or removed from the objects in an image depends on the size and shape of the structuring element used to process the image (Namdari and Salehi, 2017).

After we obtained a binary palmprint image from the previous step, we applied the Dilation process. Dilation (usually represented by $\oplus$ ) is basically developed for binary images and the dilation process often uses a structuring element for probing and increasing the shapes found in the input image (Sonka et al., 2014). The Structure Element (SE) is a simple view which regards an image with a simple, pre-defined shape and the process is used to conclude how this shape suits or misses the shapes in the image. SE is a binary image (Imani et al., 2015). In this current research, we used a $9 * 9$ matrix max for the structure element due to the large size of the palmprint images.

The dilation of $A$ by $B$ is defined as following (Sagar and Lim, 2015):

$$
A \oplus B=\bigcup_{b \in B} A_{b}
$$

where, $A_{b}$ is the translation of $A$ by $b$.

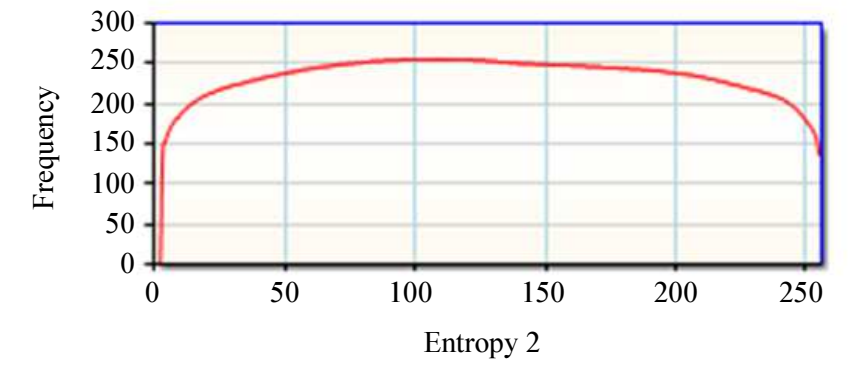

(a)

\begin{tabular}{|l|l|l|l|c|}
\hline & $\begin{array}{l}\text { Size } \\
\text { entropy }\end{array}$ & $\begin{array}{l}\text { Max } \\
\text { value }\end{array}$ & Location & Time \\
\hline$\downarrow$ & Entropy 2 & $9.155282 \ldots$ & 108 & $00: 00: 06 \ldots$ \\
\hline & & & & \\
\hline
\end{tabular}

(b)

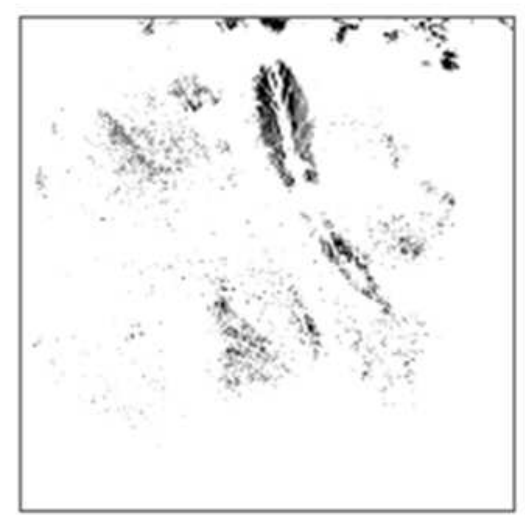

(c)

Fig. 6: Binarization steps of the palmprint image (a) Entropy calculation (b) Max entropy (c) Binery palmprint 


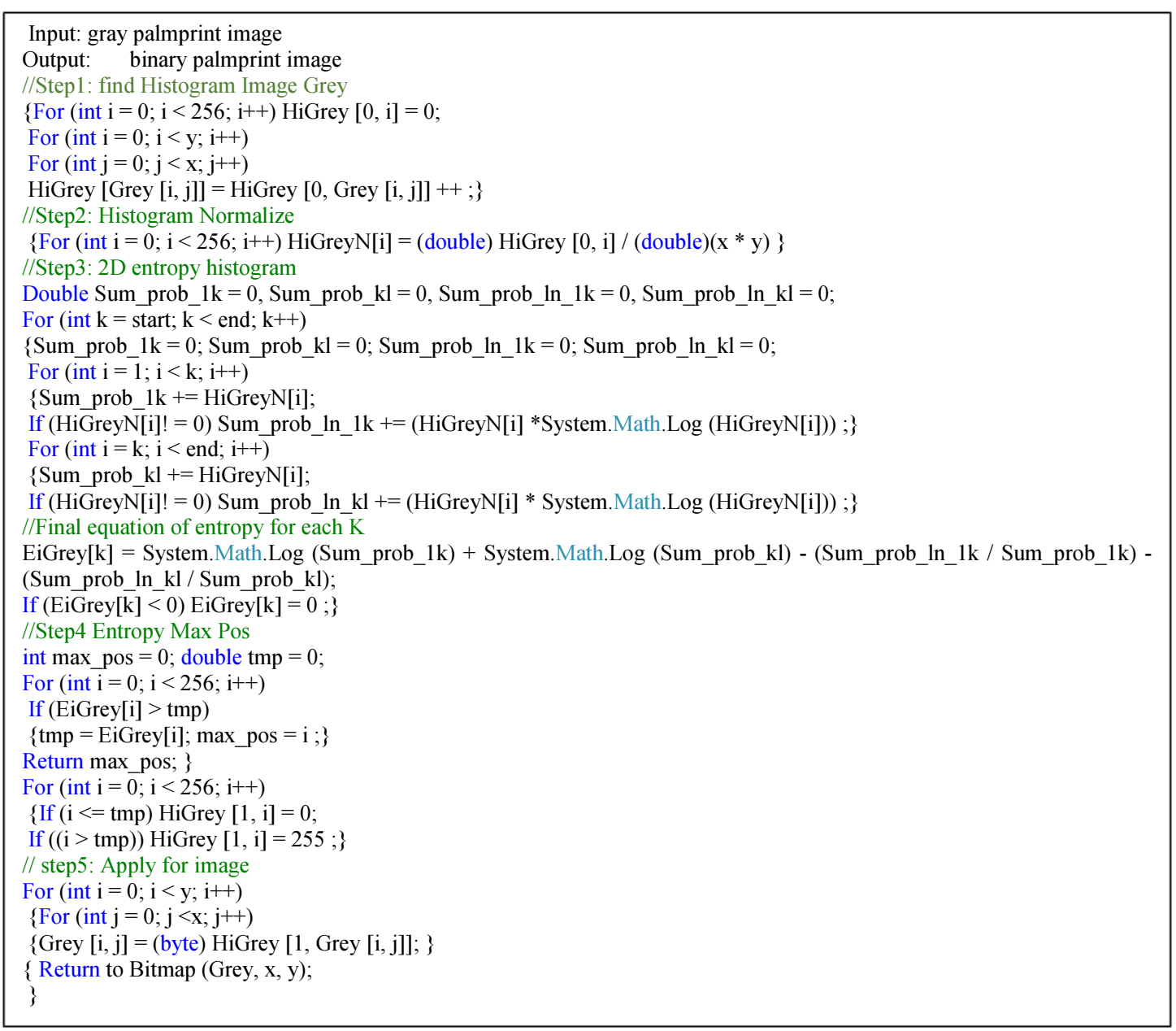

Fig. 7: Binarization pseudocode algorithm steps

The use of dilation is to find a large space on the palmprint image by gradually enlarging the boundaries of the regions of the foreground pixels. Thus, the areas of the foreground pixels grow in size while the holes within those regions become smaller in order to force the details of the image. Figure 8 shows a sample of the effect of the dilation process on the binary palmprint image.

\section{B. Construct the ROI}

The ROI is the area of an image that contains the information of interest. To extract the ROI that is in high resolution is challenging, as we mentioned earlier that the image is affected by skin distortion and unallocated images. In the current paper, we proposed the extraction of the ROI by using a novel procedure so we can deal with all these problems.

First, the dilated palmprint image was divided into four regions to remove the regions surrounding the ROI and they are named as the top, bottom left and right regions.

Second, the region of interest on the palmprint image where the reference points were situated needed to be cropped. In order to deal with the unallocated and ununiformed image, these reference points are specified based on the calculation of the percentage of the white and black points in each row and column in all four regions as shown in Fig. 9. The result is the rectangular region which is drawn based on these points. The size of these regions is different from one image to another. The following algorithm illustrates the steps of the ROI Construct:

Step 1: From the top region:

i. Calculate the white points percentage (WP\%) in each row

ii. Find $\mathrm{i} \min <=\mathrm{WP} \%$

iii. Move region top +1

iv. End

Step 2: From the bottom region:

i. Calculate the white points percentage (WP\%) in each row

ii. Find $\mathrm{i} \max <=\mathrm{WP} \%$ 
iii. Move region bottom-1
iv. End

Step 3: From the left region:

i. Calculate the white points percentage (WP\%) in each column

ii. Find $\mathrm{j} \min <=\mathrm{WP} \%$

iii. Move region left +1

iv. End

Step 4: From the right region:

i. Calculate white points percentage $(\mathrm{WP} \%)$ in each column

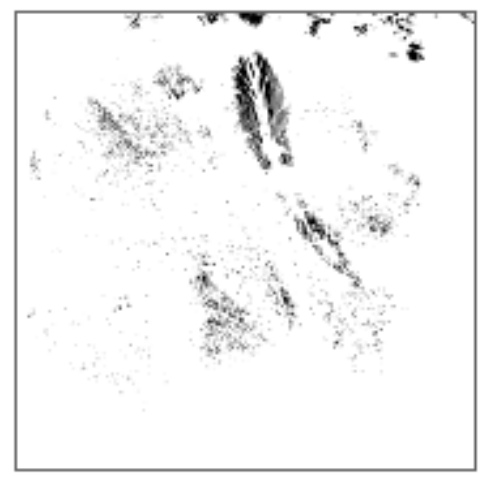

(a)
ii. Find $\mathrm{j} \max <=\mathrm{WP} \%$
iii. Move region right -1
iv. End

Step 5: Find:

- $\quad$ Rf1 = top, left (i min, j min)

- $\quad$ Rf2 = top, right (i min, j max)

- $\quad$ Rf3 = bottom, left (i max, j min)

- $\quad \mathrm{Rf} 4=$ bottom, right (i max, j max)

Step 6: Crop the ROI

Figure 10 illustrates the locations of the RF's and ROI crops on the sample of the palmprint image.

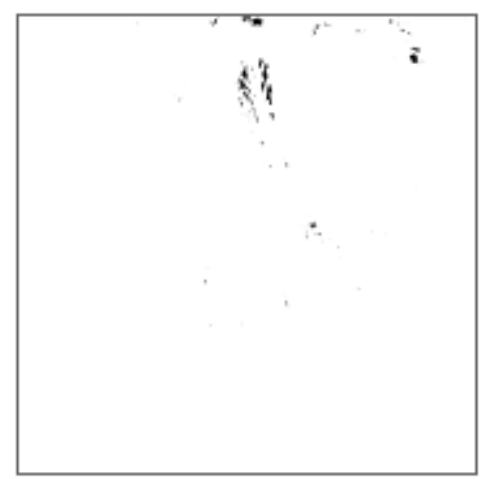

(b)

Fig. 8: Dilated palmprint image; (a) Binary image (b) Dilate image

\begin{tabular}{|c|c|c|c|c|c|c|c|c|c|c|c|}
\hline & \# & Type & Black\% & White\% & ^ & & \# & Type & Black\% & White\% & $\wedge$ \\
\hline \multirow[t]{6}{*}{ - } & 10 & Top & $3.3333 \%$ & $96.6176 \%$ & & \multirow[t]{6}{*}{ । } & 10 & Left & $49.6569 \%$ & $50.2941 \%$ & \multirow{6}{*}{$\checkmark$} \\
\hline & 11 & Top & $3.2843 \%$ & $96.667 \%$ & & & 11 & Left & $49.6569 \%$ & $50.2941 \%$ & \\
\hline & 12 & Top & $4.0686 \%$ & $95.8824 \%$ & & & 12 & Left & $49.6569 \%$ & $50.2941 \%$ & \\
\hline & 13 & Top & $3.7255 \%$ & $96.2255 \%$ & & & 13 & Left & $49.6569 \%$ & $50.2941 \%$ & \\
\hline & 14 & Top & $3.6275 \%$ & $96.3235 \%$ & & & \multirow{2}{*}{\begin{tabular}{|l}
14 \\
15 \\
\end{tabular}} & Left & $49.6569 \%$ & $50.2941 \%$ & \\
\hline & 15 & Top & $3.5784 \%$ & $96.3725 \%$ & & & & Left & $49.6569 \%$ & \multirow[t]{2}{*}{$50.2941 \%$} & \\
\hline & & & & & & & & & & & \\
\hline & \# & Type & Black\% & White\% & $\wedge$ & & \# & Type & Black\% & White\% & $\wedge$ \\
\hline \multirow[t]{6}{*}{$\vdash$} & 2039 & bottom & $99.4608 \%$ & $0.0000 \%$ & & \multirow[t]{6}{*}{ • } & 2039 & Right & $99.9510 \%$ & $0.0000 \%$ & \\
\hline & 2038 & bottom & $99.4608 \%$ & $0.0000 \%$ & & & 2038 & Right & $99.9510 \%$ & $0.0000 \%$ & \\
\hline & 2037 & bottom & $99.4608 \%$ & $0.0000 \%$ & & & 2037 & Right & $99.9510 \%$ & $0.0000 \%$ & \\
\hline & 2036 & bottom & $99.4608 \%$ & $0.0000 \%$ & & & 2036 & Right & $99.9510 \%$ & $0.0000 \%$ & \\
\hline & 2035 & bottom & $0.1471 \%$ & $99.3137 \%$ & & & 2035 & Right & $49.6569 \%$ & $50.2941 \%$ & \\
\hline & 2034 & bottom & $0.1471 \%$ & $99.3137 \%$ & v & & 2034 & Right & $49.6569 \%$ & $50.2941 \%$ & $\checkmark$ \\
\hline
\end{tabular}

(a)

\begin{tabular}{|l|l|l|}
\hline & Type & Loc \\
\hline$\downarrow$ & ReginTop & 0 \\
\hline & Reginbot... & 2036 \\
\hline & ReginLeft & 509 \\
\hline & ReginRight & 1530 \\
\hline$*$ & $\cdots$ & \\
\hline & & \\
\hline
\end{tabular}

(b)

Fig. 9: Reference points identification; (a) The percentage values of black and white points (b) RF points values 


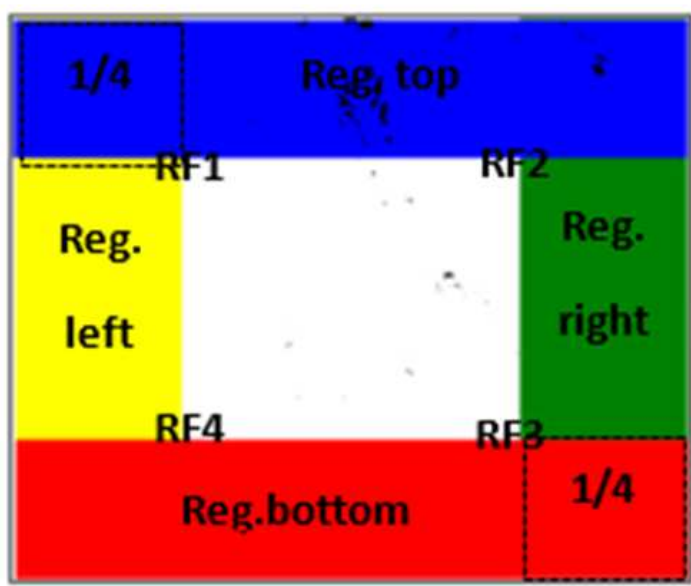

(a)

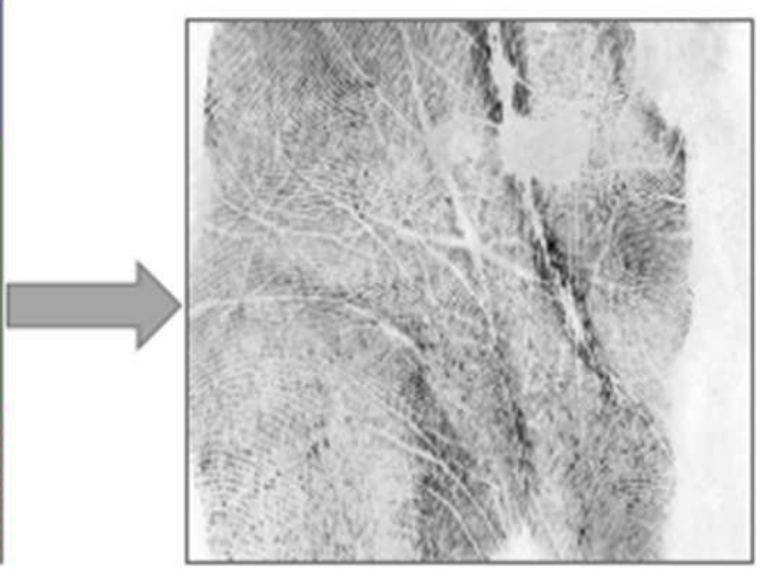

(b)

Fig. 10: The sample of the ROI palmprint (a) Four regions (b) ROI

\section{Experiment Result}

THUPALMLAB database is used in this experiment. It is the only public high-resolution palmprint image database (Fei et al., 2018). This database contains 1,280 palmprint images from 80 subjects (two palms per person and eight impressions per palm) captured using a commercial palmprint scanner of Hisign. All the palmprint images are of $2040 \times 2040$ pixels and 500 ppi (THU, 2012). We will use all images in the database $(80 \times 2 \times 8)$ to evaluate our proposed method. Figure 11 shows the samples of the THUPALMLAB database. C\# (Microsoft Visual Studio 2013) is used to implement the algorithms using Windows 10.

After determining the 2D image histogram entropy, the max entropy position was selected. Table 1 gives an example of the acquired max entropy from a sample of palmprint images. These values are used as an adaptive threshold. Each image has a different threshold which will be used to binarize the palmprint image. This calculation is not complicated and it can be accomplished in a short time.

Table 2 provides the samples of reference point values which are acquired from the four regions. As mentioned before, the choice of the reference points is based on $\mathrm{WP} \%$. In the experiment, we uncover that the $\mathrm{WP} \%=95 \%$, which gives us a good location of the ROI. Figure 12 shows the samples of the ROI palmprint which resulted from the completion of all the steps stated earlier. Figure 13 shows the sample of the miss-crop palmprint image which resulted from a complete distortion of the image. To calculate the accuracy of this approach, the calculations will be done manually by inspecting the faulty cropping (Mandeel et al., 2018):

$$
\text { Accuracyrate }=\frac{(\text { all images }- \text { fault image })}{\text { all images }}=\frac{1195}{1280} \times 100 \%=93 \%
$$

Table 1: Max entropy values for five palmprint images

\begin{tabular}{llll}
\hline Image no. & Max Entropy & Location & Time $(\mathrm{sec})$ \\
\hline 1_1_1 & 9.155288 & 108 & 10 \\
1_r_1 & 9.316268 & 130 & 09 \\
2_1_1 & 8.598335 & 99 & 09 \\
2_r_1 & 9.301394 & 133 & 09 \\
3_1_3 & 8.849170 & 112 & 10 \\
3_r_3 & 9.153842 & 130 & 09 \\
4_1_1 & 9.193457 & 128 & 09 \\
4_r_1 & 7.784664 & 176 & 08 \\
5_1_1 & 9.114948 & 108 & 09 \\
5_r_1 & 8.946113 & 129 & 10 \\
\hline
\end{tabular}

(l is left palmprint and $r$ is right palmprint)

The literature review has suggested the highresolution palmprint biometric system and we can compare the results obtained from the literature with corresponding comes from this study. However, this evaluation suffers from some limits, in particular when tests strike structured on conditions, different criteria and/or for the several database, making immediate comparisons harder. We will compare our work with an original work which was introduced by (Wang et al., 2013a) as shown in Table 3. Where (Wang et al., 2013a) proposed an algorithm to segment the palmprint image called automatic segmentation into three regions and used the last 50 subjects $(50 \times 2 \times 8)$ from the THUPALMLAB database to evaluate their work. They used the segmentation error parameter to measure the accuracy of the segmentation algorithm which is indicated in (Goumeidane and Khamadja, 2010).

Observing Table 3, it is explicit that the proposed method outperforms the original method to segment the high-resolution palmprint image. 
Inass Shahadha Hussein et al. / Journal of Computer Science 2019, 15 (5): 635.647

DOI: $10.3844 /$ jcssp.2019.635.647

Table 2: Rreference points location for five palmprint

\begin{tabular}{|c|c|c|c|c|c|}
\hline Image no. & RF & Loc. & Image no. & RF & Loc. \\
\hline \multirow[t]{4}{*}{ 1_1_1 } & Reg. top & 0 & 1_r_1 & Reg. top & 482 \\
\hline & Reg. down & 2036 & & Reg. down & 2036 \\
\hline & Reg. left & 509 & & Reg. left & 414 \\
\hline & Reg. right & 1530 & & Reg. right & 1915 \\
\hline \multirow[t]{4}{*}{ 2_1_1 } & Reg. top & 459 & 2_r_1 & Reg. top & 441 \\
\hline & Reg. down & 1542 & & Reg. down & 1781 \\
\hline & Reg. left & 186 & & Reg. left & 456 \\
\hline & Reg. right & 1677 & & Reg. right & 1714 \\
\hline \multirow[t]{4}{*}{ 3_1_3 } & Reg. top & 60 & 3_r 3 & Reg. top & 274 \\
\hline & Reg. down & 1755 & & Reg. down & 1874 \\
\hline & Reg. left & 296 & & Reg. left & 447 \\
\hline & Reg. right & 1830 & & Reg. right & 1759 \\
\hline \multirow[t]{4}{*}{ 4_1_1 } & Reg. top & 101 & 4_r_1 & Reg. top & 261 \\
\hline & Reg. down & 2036 & & Reg. down & 2036 \\
\hline & Reg. left & 509 & & Reg. left & 509 \\
\hline & Reg. right & 1533 & & Reg. right & 1617 \\
\hline \multirow[t]{4}{*}{ 5_1_1 } & Reg. top & 299 & 5_r_1 & Reg. top & 498 \\
\hline & Reg. down & 1893 & & Reg. down & 2000 \\
\hline & Reg. left & 113 & & Reg. left & 509 \\
\hline & Reg. right & 2009 & & Reg. right & 2023 \\
\hline
\end{tabular}

(l is left palmprint and $\mathrm{r}$ is right palmprint)

Table 3: Comparison between the proposed method and automatic segmentation method

\begin{tabular}{lcll}
\hline Work & Number of images & Successful segmented images & Parameter \\
\hline Wang et al. (2013a) & 800 & 702 & Total error rate $=19.54 \%$ \\
Proposed work & 1280 & 1195 & Accuracy $=93 \%$ \\
\hline
\end{tabular}

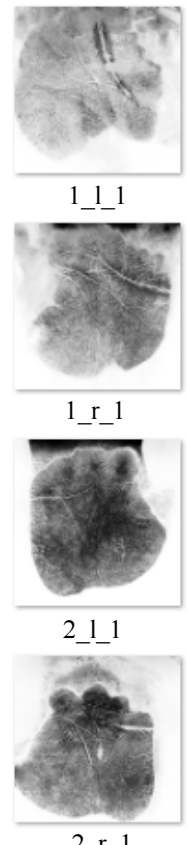

2_r_1

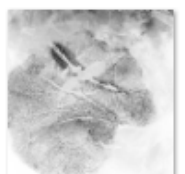

1_1_2

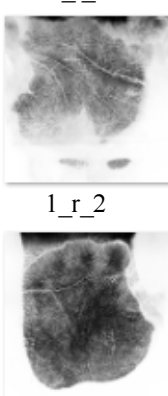

2_1_2

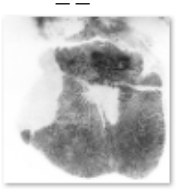

2_r_2

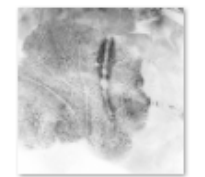

1_1_3
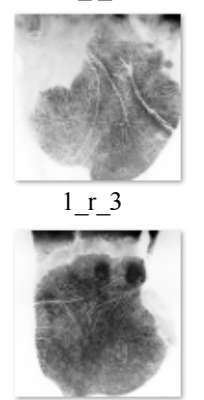

2_1_3

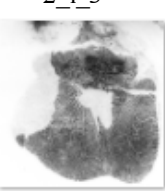

2_r_3

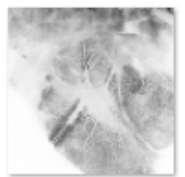

1_1_4
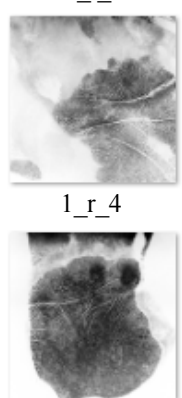

2_1_4

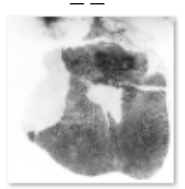

2_r_4

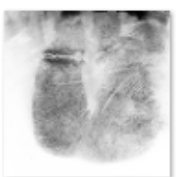

1_1_5

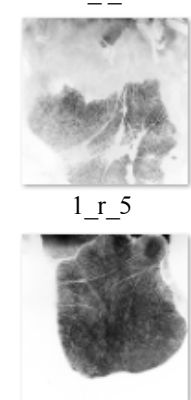

2_1_5

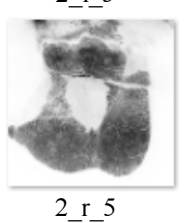

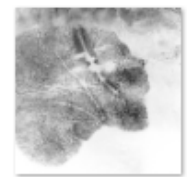

1_1_6

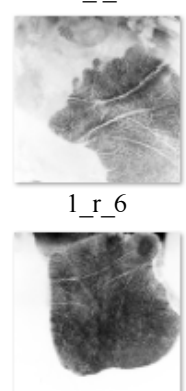

2_1_6

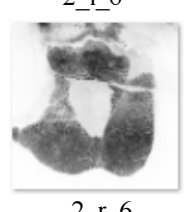

2_r 6

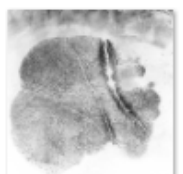

1_1_7

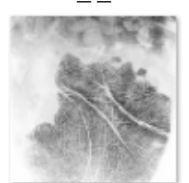

1_r 7

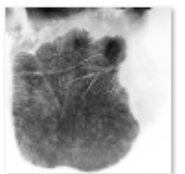

2_1_7

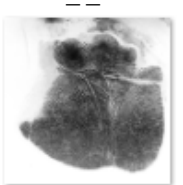

2_r_7

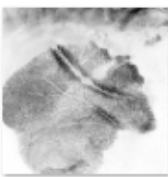

1_1_8

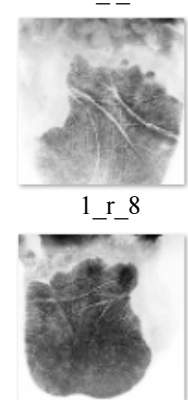

2_1 8

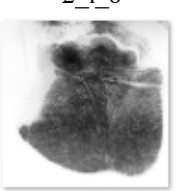

2_r_8

Fig. 11: Samples of THUPALMLAB database 

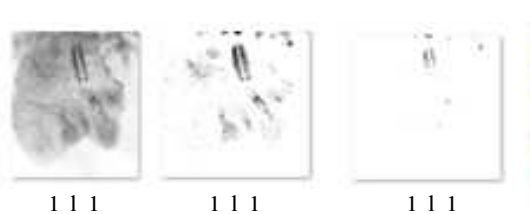

1_1_1
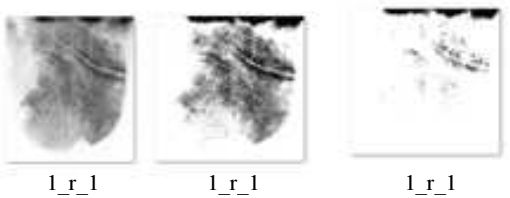

1_r_1

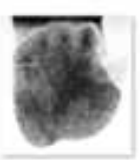

2_1_1
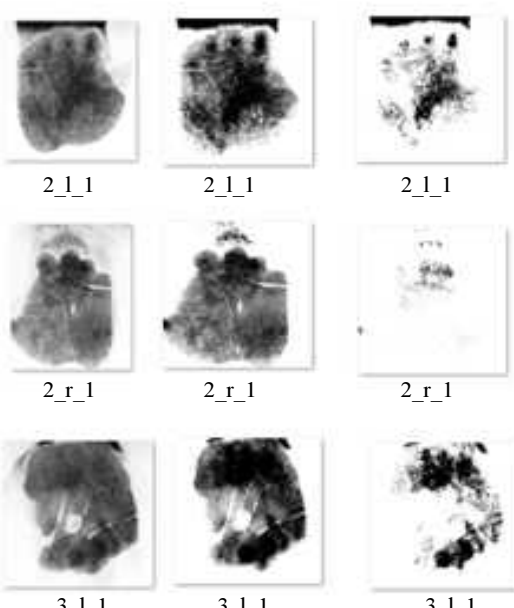

3_1_1
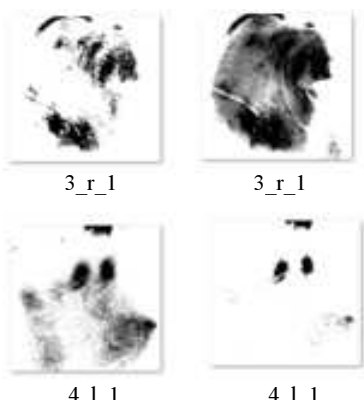

4_1_1
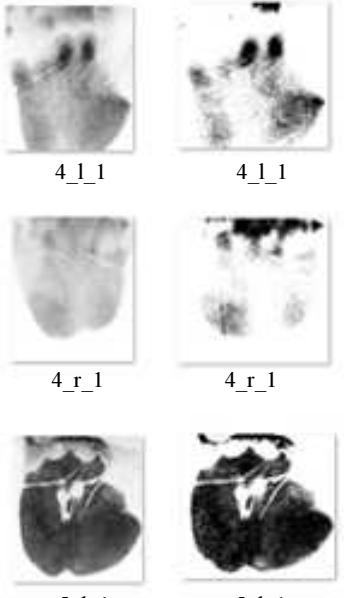

5_1_1
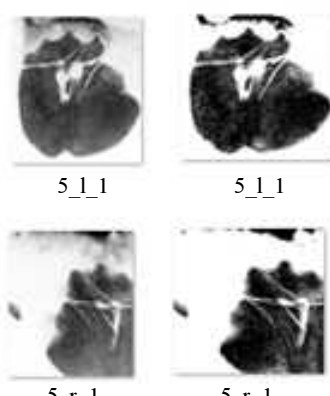

(a)

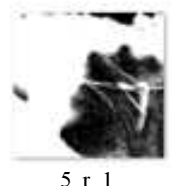

(b)
,

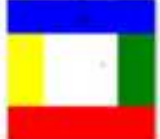

111
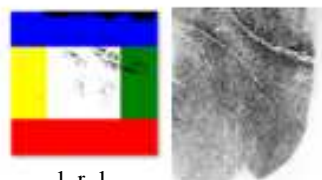

$1 \_r \_1$
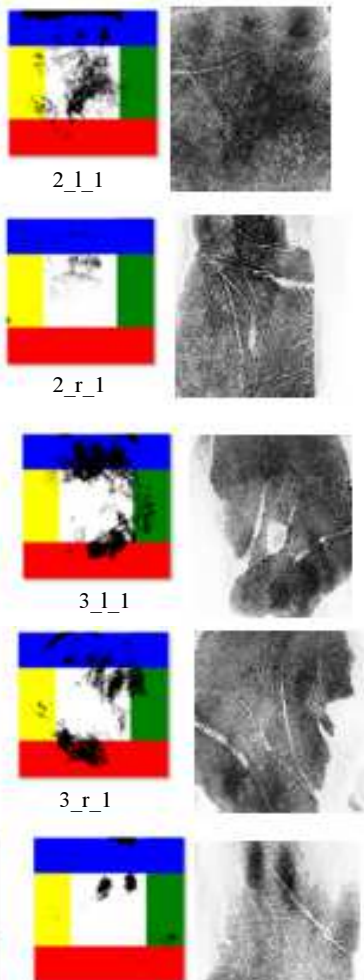

4_1_1
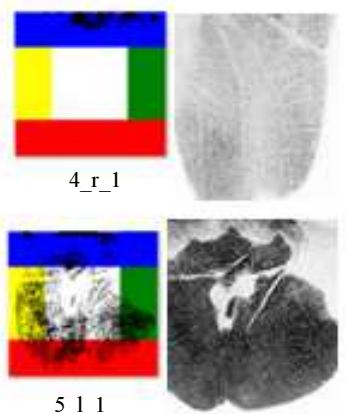

51

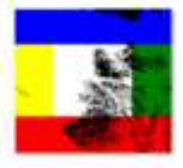

$5 \mathrm{r}$

(d)

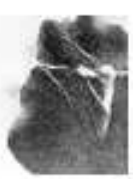

(e)

Fig. 12: The proposed method's steps (a) Original image (b) Binerzation image (c) Dilated image (d) Four region (e) ROI image 


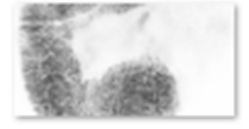

17 1_1

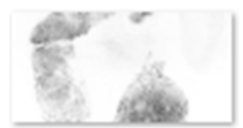

154_1_1

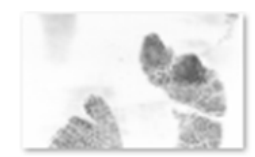

45 r_1

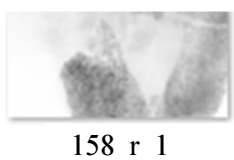

$158 \_$r_1

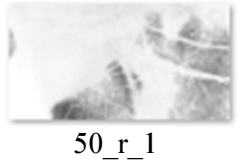

50_r_1

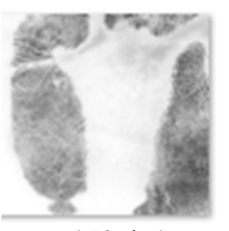

15911

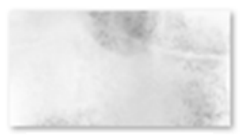

$62 \_r+1$

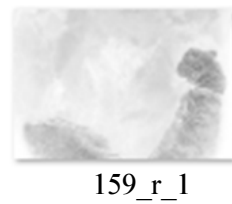

Fig. 13: Samples of fault ROI palmprint

\section{Conclusion}

Although the high-resolution palmprint images are important in the forensic application, there has been a very little attention to these images investigation. Therefore, this study investigates a new method for the high-resolution palmprint images segmentation to extract the ROI in order to be used in the recognition system. Undoubtedly, the image of ROI extraction plays a major role in the performance of any biometric-based recognition system. The proposed method is an attempt to find a suitable segmentation for the high-resolution palmprint image. The problems with these images are that they are distorted and varied in location, so it is difficult to find the perfect ROI. Our method includes two phases; binarization image phase and ROI extraction phase. The binarization phase includes; first, removing noise by using the median filter. Second, determining the 2D image histogram entropy. And third, we adopt the max entropy as an adaptive threshold, that is utilized to binarize the image as an effective method. Furthermore, the ROI phase includes; first, dilating the binary image by using the morphology-dilation which helps to increase the details of the images. Second, the region of interest is constructed by dividing the dilated image into four regions and calculating the percentage of the white points in each region in order to specify reference points based on these percentages which are adaptable according to the different images in the database. The proposed method is able to extract ROI more consistently. When the images are significantly distorted, the results will be regarded as the missing-cropped ROI. The accuracy of this work is $93 \%$. We proposed that this study will help improve high-resolution palmprint recognition.

\section{Acknowledgment}

The researchers wish to thank Universiti Teknologi Malaysia (UTM) and Universiti Kebangsaan Malaysia (UKM) for supporting this work by awarding a research grant DIP-2016-018.

\section{Authors Contribution}

Shamsul Bin Sahibuddin, Md Jan Nordin and Nilam Nur Amir Sjarif: Supervising, providing the research topic, reviewing and editing.

Inass Shahadha Hussein: Methodology, experiment and writing.

\section{Ethics}

This paper is an original work which could be published without any ethical issues.

\section{References}

Ashtari, A.H., M. J. Nordin, S. M. M. Kahaki. 2015. Double line image rotation. IEEE Transactions on Image Processing 24(11):3370-3385.

Brusius, F., U. Schwanecke and P. Barth, 2011. Blind image deconvolution of linear motion blur. Proceedings of the International Conference on Computer Vision, Imaging and Computer Graphics, Mar. 5-7, Springer, Vilamoura, Portugal, pp: 105-119.

Carreira, L., P.L. Correia and L.D. Soares, 2014. On high resolution palmprint matching. Proceedings of the 2nd International Workshop on Biometrics and Forensics, Mar. 27-28, IEEE Xplore Press, Valletta, Malta, pp: 1-6.

DOI: 10.1109/IWBF.2014.6914247.

Fei, L., G. Lu, W. Jia, S. Teng and D. Zhang, 2018. Feature extraction methods for palmprint recognition: A survey and evaluation. IEEE Trans. Syst. Man Cybernet.: Syst., 49: 346-363. DOI: 10.1109/TSMC.2018.2795609

Gonzalez, R.C. and R.E. Woods, 2002. Digital Image Processing. 1st Edn., Prentice Hall Upper Saddle River, NJ.

Goumeidane, A.B. and M. Khamadja, 2010. Error measures for segmentation results: Evaluation on synthetic images. Proceedings of the 17th IEEE International Conference on Electronics, Circuits and Systems, IEEE. 
Hussein, I.S. and M.J. Nordin, 2014. Palmprint verification using invariant moments based on wavelet transform. J. Comput. Sci., 10: 1389. DOI: $10.3844 /$ jcssp.2014.1389.1396.

Imani, E., M. Javidi and H.R. Pourreza, 2015. Improvement of retinal blood vessel detection using morphological component analysis. Comput. Meth. Programs Biomed., 118: 263-279. DOI: $10.1016 /$ j.cmpb.2015.01.004.

Jain, A.K. and J. Feng, 2009. Latent palmprint matching. IEEE Trans. Patt. Anal. Mach. Intell., 31: 1032-1047. DOI: 10.1109/TPAMI.2008.242.

Jamshidnezhad, A. and M.J. Nordin, 2012. A heuristic model for optimizing fuzzy knowledge base in a pattern recognition system. J. Scientific Industrial Res., 71: 341-347.

Jaswal, G., A. Kaul and R. Nath, 2017. Palm print ROI extraction using Bresenham line algorithm. Proceedings of the 4th International Conference on Signal Processing, Computing and Control, Sept. 2123, IEEE Xplore Press, Solan, India, pp: 547-552. DOI: 10.1109/ISPCC.2017.8269739.

Kahaki, S.M.M., M. J. Nordin, A. H. Ashtari, S. J. Zahra. 2016. Deformation invariant image matching based on dissimilarity of spatial features. Neurocomputing, 175:1009-1018.

Kim, H., E. Ahn, S. Cho, M. Shin and S.H. Sim, 2017. Comparative analysis of image binarization methods for crack identification in concrete structures. Cement Concrete Res., 99: 53-61.

DOI: 10.1016/j.cemconres.2017.04.018.

Kim, M.K., 2011. Palmprint recognition based on line and slope orientation features. J. Inform. Sci. Eng., 27: 1219-1232.

Kumar, A., 2018. Toward more accurate matching of contactless palmprint images under less constrained environments. IEEE Trans. Inform. Forens. Security, 14: 34-47. DOI: 10.1109/TIFS.2018.2837669

Mandeel, T.H., M.I. Ahmad, M.N.M. Isa, S.A. Anwar and R. Ngadiran, 2018. Palmprint region of interest cropping based on Moore-neighbor tracing algorithm. Sens. Imag., 19: 15-15. DOI: $10.1007 / \mathrm{s} 11220-018-0199-6$

Mousavi Kahaki, S.M., M.J. Nordin, A.H. Ashtari and S.J. Zahra. 2016. Invariant feature matching for image registration application based on new dissimilarity of spatial features. PLoS One.

Namdari, F. and M. Salehi, 2017. High-speed protection scheme based on initial current traveling wave for transmission lines employing mathematical morphology. IEEE Trans. Power Delivery, 32: 246-253. DOI: 10.1109/TPWRD.2016.2571341

Nie, F., P. Zhang, J. Li and D. Ding, 2017. A novel generalized entropy and its application in image thresholding. Signal Process., 134: 23-34.

DOI: 10.1016/j.sigpro.2016.11.004.
Sagar, B.D. and S.L. Lim, 2015. Ranks for pairs of spatial fields via metric based on grayscale morphological distances. IEEE Trans. Image Process., 24: 908-918. DOI: $10.1109 /$ TIP.2015.2390135

Sezgin, M. and B. Sankur, 2004. Survey over image thresholding techniques and quantitative performance evaluation. J. Electr. Imag., 13: 146-166. DOI: $10.1117 / 1.1631315$.

Sonka, M., V. Hlavac and R. Boyle, 2014. Image processing, analysis and machine vision. Cengage Learning.

THU, 2012. THU 500PPI palmprint database.

Wang, R., D. Ramos and J. Fierrez, 2012. Improving radial triangulation-based forensic palmprint recognition according to point pattern comparison by relaxation. Proceedings of the 5th IAPR International Conference on Biometrics, Mar. 29Apr. 1, IEEE Xplore Press, New Delhi, India, pp: 427-432. DOI: 10.1109/ICB.2012.6199788

Wang, R., D. Ramos, J. Fierrez and R.P. Krish, 2013a. Automatic region segmentation for high-resolution palmprint recognition: Towards forensic scenarios. Proceedings of the 47th International Carnahan Conference on Security Technology, Oct. 8-11, IEEE Xplore Press, Medellin, Colombia, pp: 1-6. DOI: 10.1109/CCST.2013.6922078.

Wang, R., D. Ramos, J. Fierrez and R.P. Krish, 2013b. Towards regional fusion for high-resolution palmprint recognition. Proceedings of the 26th SIBGRAPIConference on Graphics, Patterns and Images, Aug. 58, IEEE Xplore Press, Arequipa, Peru, pp: 357-361. DOI: 10.1109 /SIBGRAPI.2013.56

Wang, R., D. Ramos, R. Veldhuis, J. Fierrez and L. Spreeuwers et al., 2014. Regional fusion for highresolution palmprint recognition using spectral minutiae representation. IET Biometr., 3: 94-100. DOI: $10.1049 /$ iet-bmt.2013.0067.

$\mathrm{Wu}, \mathrm{B}$., H. Zeng and $\mathrm{H}$. $\mathrm{Hu}, 2018$. Illumination invariant feature point matching for high-resolution planetary remote sensing images. Planetary Space Sci., 152: 45-54. DOI: 10.1016/j.pss.2018.01.007.

Yang, K., R. Yu, X. Wang, M. Quddus and L. Xue, 2018. How to determine an optimal threshold to classify real-time crash-prone traffic conditions? Accident Anal. Prevent., 117: 250-261. DOI: 10.1016/j.aap.2018.04.022.

Zhang, L., L. Li, A. Yang, Y. Shen and M. Yang, 2017. Towards contactless palmprint recognition: A novel device, a new benchmark and a collaborative representation based identification approach. Patt. Recognit., 69: 199-212.

DOI: 10.1016/j.patcog.2017.04.016

Zhang, S., H. Wang, W. Huang and C. Zhang, 2018. Combining modified LBP and weighted SRC for palmprint recognition. Signal Image Video Process., 12: 1035-1042. DOI: 10.1007/s11760-018-1246-4 